\title{
The Vehicle Speed Detection Algorithm of Virtual Bayonet System
}

\author{
Xue Lixia $^{1, a}$, LuoYanli ${ }^{2, b}$, Guo Qingbin ${ }^{3, c}$ \\ ${ }^{1}$ Computer College, Hefei University of Technology, Hefei, Anhui 230009, China \\ ${ }^{2}$ Enterprise Technology Center, Anhui Sun Create Electronic Co., LTD, Hefei, Anhui 230088, China \\ ${ }^{3}$ System Integration Center, Anhui ZXJY Information Technology Co., LTD, Hefei Anhui 230031,China. \\ a51003239@qq.com, b442590198@qq.com, ${ }^{\text {c} 409033212 @ q q . c o m ~}$
}

\begin{abstract}
With the deepening of the "safe city" construction, to introduce a large number of high-definition cameras video data as the foundation, construction of the virtual bayonet system. The system uses image processing technology for real-time monitoring of the movement of vehicles, the use of moving vehicle detection and tracking methods to achieve the speed of the moving vehicle detection; Provide traffic parameter for the safe city system, realize the city's network surveillance, to provide effective information for the public security case investigation, improves the automation of a business system of public security.
\end{abstract}

Keywords—component; formatting; style; styling; insert

\section{INTRODUCTION}

With the deepening of the construction of "safe city", the introduction of high-definition camera number has increased dramatically, on the basis of high-definition video camera data construction of the virtual bayonet system. And video motion speed of the vehicle is one of the important parameters of the virtual bayonet system, is to realize the urban network monitor and improve the basis of public security business system automation.

In this paper, vehicle speed detection of virtual bayonet system is to use the road high-definition camera and digital image processing method for video image sequence analysis and processing, Using background difference method and frame difference method combined with vehicle moving target detection in video sequences, Motion vehicle detection with the actual the change of the external factors such as illumination in the scene, scene background will often change, so the background image needs to updated in real time, using mathematical morphological operation to merge noise field and eliminating scattered holes in the target area, and then extract the complete moving targets ;Using the extended Kalman filter fast target tracking algorithm to detect the vehicle target tracking, to extract the vehicle target location information. According to the camera calibration method to obtain the image displacement and movement distance of the vehicle; Using frames timing method to obtain the interval between frames, Actual speed of the vehicle is detected according to the formula.

\section{ALGORITHM IMPLEMENTATION}

In this paper, the vehicle speed detection algorithm of virtual bayonet system, mainly includes moving vehicles target detection, vehicle moving target tracking and vehicle speed detection, the algorithm flow chart is as follows.

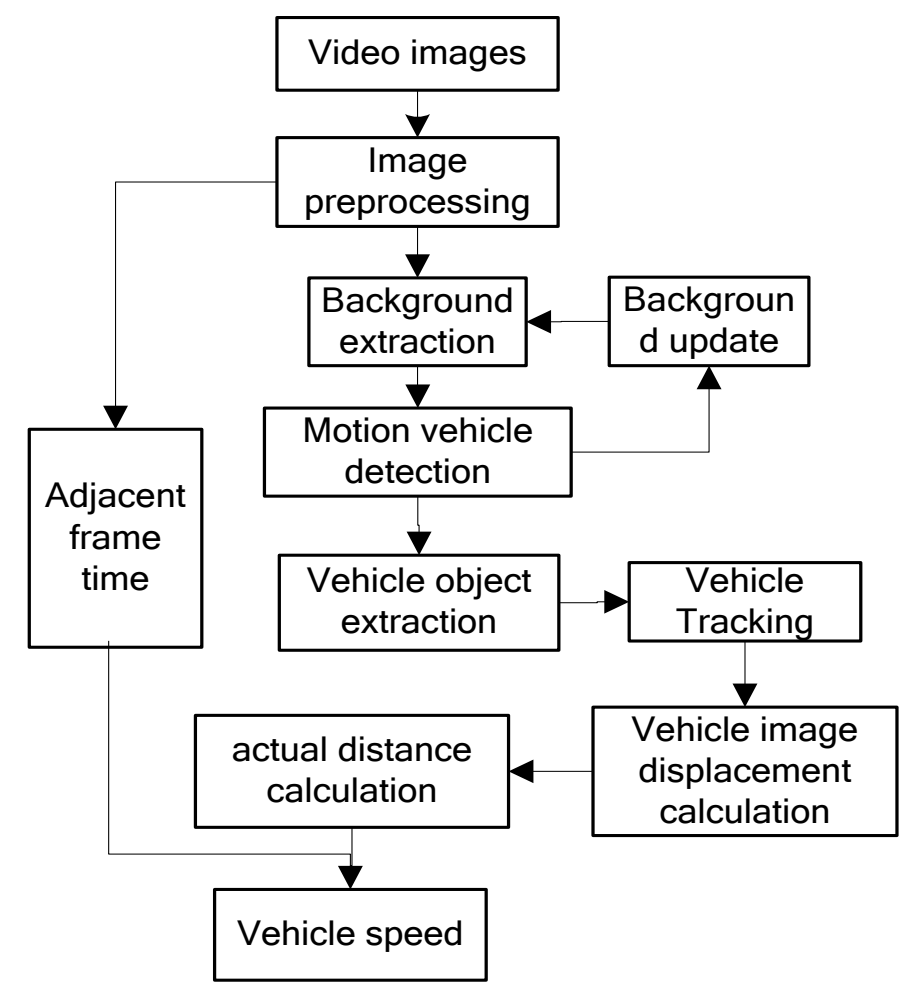

Fig. 1. Algorithm flow figure

\section{Moving Vehicle Detection}

The video sequence image preprocessing, Average method is used to extract the background image, and the adaptive background updating method is adopted for background update, through difference method and frame difference method Combination accurate way to accurately extract the current frame the movement of the vehicle. 
- $\quad$ video sequence frame image extraction intervals, To gray-scale image processing, using average method to extract the initial background image ;

- The grayscale of the and the frame image of the video sequence at the point are , ; the frame difference method to obtain change area image , the background difference method to obtain the initial target image ;

- The real-time dynamically updating background. Background difference method to extract the moving object, is sensitive to the change of the background, when the background illumination changes, if not timely update the background image will produce error detection results, so for a long time to the detection of target, background light is necessarily change over time, must adopt the method of adaptive background updating;

- Moving region Image and initial target image is AND operation to obtain the initial motion silhouette target image ; initial target image and motion silhouette target image is XOR to obtain empty area image ; initial target image and empty area image is OR to obtain moving vehicle image ;

- Using mathematical morphology operation to moving vehicle image of sports field and eliminate the target holes, and then extract the complete moving targets.

\section{Moving Vehicle Tracking}

According to the detected moving vehicle, using the extended Kalman filter algorithm for fast tracking the target vehicle tracking, at the same time, to extract the vehicle target location information.

- According to the detected moving vehicles, establish moving target tracking window;

- Start of the first track of the frame image, to the image of the detected motion of the vehicle target established Kalman filter, and the respective parameters are initialized;

- According to the motion of the vehicle target detection, access to the vehicle centroid location, size and other characteristics of the vehicle value, and according to these characteristics established using the extended Kalman filter motion model to predict the target position in the next frame of the vehicle that may arise;

- Using extended Kalman filter in the next frame the prediction range to search target images, and in accordance with the rules of the moving object matching;

- Target matching success is moving target template updating, if does not match with new target templates to create;

- Accurate vehicle moving target tracking, and to extract the vehicle tracking position information.

\section{SPEED DETECTION}

Based on vehicle tracking for vehicle target position in different frames of image information, using the camera calibration method for vehicle image displacement and the actual relative displacement transformation, Obtained from the motion of the vehicle, Using frames timing method to obtain the interval between frames the time interval between adjacent frames are known, the speed of the vehicle is obtained according to the formula.

- when the number of frames timing determination of traffic time, this method refers to some frames in video sequences in the image acquisition card to moment;

- When the video sequence is a frame in the vehicle to begin moving vehicle detection, and the interval between 15 frames for the fixed time tracking the vehicle target, according to the tracking record vehicle target position in different frames of image points and image acquisition time;

- According to the camera calibration and vehicles target in the position of the image coordinates of point target , to get the vehicle target in the actual physical three-dimensional coordinate position, the vehicle target position on an actual road;

- According to the multiple locations on the vehicle target in the actual path points to calculate the vehicles in the segment time distance;

- According to the formula to calculate the vehicle speed.

\section{CONCLUSION}

Vehicle speed detection algorithm of virtual bayonet system, this paper uses the high-definition cameras installed on road resources, using image processing method for image sequences in the video analysis and processing, detect the moving vehicle target, using an extended Kalman filter tracking method tracking, and get a vehicle target position in different frames of image information, use the camera calibration method to calculate the vehicle's movement distance, the interval between frames are known, the speed of the vehicle calculated according to the formula. The experiments show that the detection system has a reliable and stable detection accuracy can meet the requirements of realtime system, the vehicle speed detecting bayonet system.

\section{ACKNOWLEDGMENT}

This work is partially supported by the Fundamental Research Funds for the Central Universities of China (JZ2014HGBZ0059).

\section{References}

[1] Zhu Liying, Liang Chen. Surveillance video retrieval method based on moving object [D] Computer Applications and Software. Vol.28 No.12.

[2] Liu Zhiyu. Intelligent traffic detection system, dynamic target detection method [J] Computer Applications and Software, 2010, 27 (1): 29-57. 
[3] Zhai Haiting, Wu Xiao Juan, Han Huai. Research of a real-time hand tracking algorithm[C] IEEE International Conference on Neural Networks and Brains, 2005, 2(0):1233-1235.

[4] Tang Zhen, Huang Yeliang, Yang Hua. Definition Bayonet intelligent information security identification system and application [J], Television Technology, 2012, 36 (14).

[5] Kalal Z, Mikolajczyk K, Matas J. Tracking learning detection[J].Pattern Analysis and Machine Intelligence, IEEE Transactions on,2012,34(7):1409-1422.
[6] Qi Meibin, Xuan Ke, Jiang Jianguo. Algorithm based on traffic statistics contrast distortion parameters [J], Hefei University of Technology: Natural Science News, 2010, 33 (12): 1815-1823.

[7] Zhang Haiqing, Li Houqiang. Target tracking based on Monte Carlo method [J]. China Journal of Image and Graphics, 2008, 13(5):937-938.

[8] Xu Yang, Wu Chengdong, Chen Dongyue. Algorithm based on video images reviewed Automatic Incident Detection [J] ,Application Research of Computers, 2011,28 (4):1206-1210. 